\title{
Mixed Finite Element Formulation for Magnetic Fluid Oil Flow in Electromagnetic Field
}

\author{
Phey Hoon Tan $^{* 1}$ and Airil Yasreen Mohd Yassin ${ }^{1}$ \\ ${ }^{1}$ Department of Civil and Environmental Engineering, Universiti Teknologi PETRONAS,32610 Seri Iskandar, Perak Darul Ridzuan \\ Malaysia.
}

\begin{abstract}
Pressure depletion and high viscosity of crude oil in oil reservoir are the main challenges in oil recovery process. A potential solution is to employ electromagnetic heating coupled with magnetic fluid injection. The present work delivers a fundamental study on the interaction between magnetic fluid flow with electromagnetic field. The two-dimensional, incompressible flow is solved numerically using mixed finite element method. The velocity fields, temperature and pressure are the variables of interest, to be obtained by solving mass, momentum and energy equations coupled with Maxwell' equations. The fluid stress arises simultaneously with the external magnetic force which mobilises and increases the temperature of the oil flow. Verification is made against available data obtained from different numerical method reported in literature. The results justify feasibility of the mixed finite element formulation as an alternative for the modelling of the magnetic fluid flow.
\end{abstract}

\section{Introduction}

Oil recovery in reservoir has become a mainstream of focus over the past decades. Various technologies associated with oil extraction have been developed to support energy source in the existing and future oil well. There are three extraction phases categorised as primary, secondary and tertiary recovery. Primary and secondary recovery uses conventional methods such as thermal heating, water flooding and carbon dioxide injection [1]. Because of the highly viscous properties of the crude oil flowing after the secondary recovery phase, the mobility of the fluid is greatly reduced and thus requires alternative treatment. As such, the tertiary recovery phase is introduced, also known as Enhance Oil Recovery (EOR) stage which utilises nanotechnology in oil production. The idea of EOR process is carried out by injecting the nanofluids into the reservoir channel to mobilize the oil residual by altering the surface tension between water and oil in the porous medium [2].

Nanofluid is a synthesized fluid, which exhibit superiors heat transfer performance and high adaptability in various oil and gas operations [1,2]. Ferrofluid, a type of nanofluids, is made up of non-magnetic bases fluids and dispersed iron nanoparticle of diameter lesser than $100 \mathrm{~nm}$ with a surfactant layer coat. According to [3], the layer contributes to the stability of the nanofluids in the enhancement of the thermal conductivity. As proposed by [4], these nanofluids contain metallic particle that give rise to significant thermal conductivity, which are approximately three times higher compared to the conventional fluids. Low concentration mixture of ferrofluids in crude oil permits the formation of efficient energy heat transfer medium and thus leads to a more cost-effective recovery process. [5] developed a model that describes the relationship between the energy dissipation of polarizable fluid and applied magnetic field for the enhancement of heat transfer. Their study highlights the importance of ferrofluids in industry applications. The magnetization of ferrofluids is also dependent on the temperature and in such property, it gives an effective energy conversion in heat transport system [6]. The inherent magnetization characteristic of the ferrofluids allows manoeuvre to take place via external magnetic field and. Hence interaction of nanofluids with electromagnetic occurs. In the absence of magnetic field the nanoparticles are scattered. Exposing ferrofluids to external magnetic field causes these nanoparticles to align in a direction and create a stronger bond, therefore the magnetization of ferrofluids is multiplied. This alignment provides a novel method to deliver thermal energy efficiently known as Neel relaxation [7]. The excited ferrofluids are aligned and used to supply heat to a specific location in reservoir. Such a relaxation will induce a higher heating rate in flowing fluids than static fluids. [8] believes that the excited ferrofluids has the potential to mobilize the traps oil in well under quasi static motion of fluids. [9] used single phase miscible ferrofluid to mobilize the underground flow using magnetic guidance. The numerical simulation taken into consideration of strong magnetic field. However, the interaction of the magnetic fluids with electromagnetic is not well addressed. The interaction of ferrofluids can further been explored with ferrohydrodynamic (FHD) numerical analysis.

* Corresponding author: suzannetan90@gmail.com 
Numerous numerical procedures had been proposed in the past, such as Finite Volume Method(FVM), Finite Difference Method (FDM), Finite Element Method (FEM), and Lagrange multiplier. Among the methods, the most widely used is the FDM which is combined with stream function vorticity formulation due to the simplicity of algorithm. In early study of FHD principle, [10] developed a model which dealt with FHD coupled with uniform electromagnetic field. The mathematical model on a rectangular domain had been employ and solved using SIMPLE algorithm. In addition, spatially varying magnetic field had been studied using FDM and pressure-linked psuedotransient method on grid method [11-13]. As a result, dominant flow form was observed nearby the magnetic source with no evidence of secondary flow effect. [14] extended the work of [13] on hydrodynamic model, where a fully conservation system is taken into consideration, whereby they used Lorentz and magnetization force and solved using FVM. One of the major advantages of using FVM is that they are easily formulated to allow for unstructured mesh. However, in present study, Lorentz force is insignificant because of the magnetic fluids oil flow has low conductivity where it is only affected by magnetization. Improvement of the [14] model is carried out by adopting Boundary Element Method using Laplacian term, which automatically satisfy continuity term and resulting in a smaller sized algebraic equation [15]. This algorithm uses comparatively less computer resources. Computational results revealed a vortex enlarged formed near to the region of magnetic source and followed with an increase in magnetic intensity. Furthermore, the fluid flow temperature increases with the increase of intensity of magnetic field. High intensity magnetic field leads to the domination of the magnetic gradient term in the system and causes the solution instable. This turmoil can be overcome by FEM as it is a well-developed approach to achieve higher order accuracy with a fine meshing compared with FDM and FVM. The result of velocities and temperature are studied considering the influence of magnetic field.

Current study focuses on development of the numerical algorithm used to simulate the ferrohydrodynamic flow in a rectangular channel with the disturbance of external magnetic field. Present study has developed a hypothesis stating that by exciting the ferrofluids with external magnetic, additional displacement will be induced which is going to mobilize the magnetic oil flow and enhanced the recovery.

\section{Governing Equations}

Employing Boussinesq approximation, the governing equation for conservation of mass, momentum and energy in non-dimensional form for a steady laminar flow can be expressed as follows [11]:
Continuity :

$$
\frac{\partial u}{\partial x}+\frac{\partial v}{\partial y}=0
$$

Momentum :

$$
\begin{aligned}
& u \frac{\partial u}{\partial x}+v \frac{\partial u}{\partial y}+\frac{\partial P}{\partial x}-\frac{1}{\operatorname{Re}}\left(\nabla^{2} u\right)-M n_{F} T H \frac{\partial H}{\partial x}=0 \\
& u \frac{\partial v}{\partial x}+v \frac{\partial v}{\partial y}+\frac{\partial P}{\partial y}-\frac{1}{\operatorname{Re}}\left(\nabla^{2} v\right)-M n_{F} T H \frac{\partial H}{\partial y}=0
\end{aligned}
$$

Energy :

$$
\begin{aligned}
& u \frac{\partial T}{\partial x}+v \frac{\partial T}{\partial y}-\frac{1}{\operatorname{Pr} \operatorname{Re}}\left(\nabla^{2} T\right) \\
& -M n_{F} E c H(\varepsilon-T)\left(u \frac{\partial H}{\partial x}+v \frac{\partial H}{\partial y}\right)= \\
& \frac{E_{c}}{\operatorname{Re}}\left[2\left(\frac{\partial u}{\partial x}\right)^{2}+2\left(\frac{\partial v}{\partial y}\right)^{2}+\left(\frac{\partial v}{\partial x}+\frac{\partial u}{\partial y}\right)^{2}\right]
\end{aligned}
$$

where $u, v, p, T$ are the $x$ and $y$ velocity components, pressure and temperature of the fluid, respectively. The magnitude of magnetic field intensity $H$ of the magnetic field is given by [12]

$$
H(x, y)=\frac{b}{\sqrt{(x-a)^{2}+(y-b)^{2}}}
$$

where $a$ and $b$ are the position of the applied magnetic field.

The new dimensionless parameter is magnetic number $\mathrm{Mn}_{\mathrm{f}}$ can be written as:

$$
M n_{F}=\frac{h^{2} B M}{\mu^{2} \rho}
$$

where $B$ and $M$ are magnetic induction and magnetization, respectively.

\subsection{Boundary Conditions}

The inflow is assumed to be fully developed with parabolic profile in $x$-direction whereas the temperature has a linear profile. No slip condition is imposed for the velocities on upper and lower plate of channel. The pressure outlet and outflow conditions are normal derivatives taken to be zero. Fig. 1 shows the arrangement of boundary conditions and the magnetic device is assumed to be permanent magnet with nonuniform distribution. 


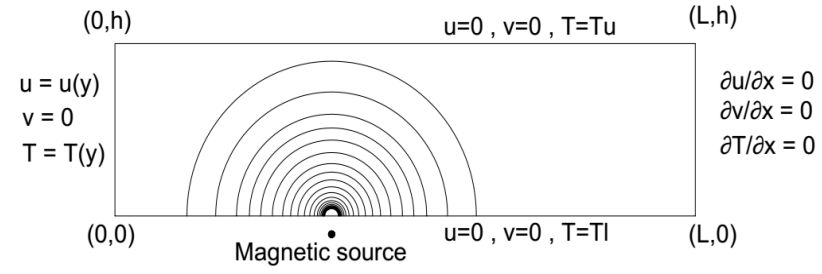

Fig. 1. Boundary conditions and magnetic field intensity.

\section{Numerical Method}

The system of partial differential equations (strong form) is discretized using Bubnov-Galerkin weighted residual method. The weighing function is taken to be the same as the interpolation function. This allows discretization of the nonlinear PDE's to obtain algebraic equations where is applicable to solve using computer programming. The stabilized formulation can be achieved by proper selection of interpolation function for velocity and pressure. The mixed interpolation means the order of the interpolation of velocity must higher than the pressure. Therefore, quadratic shape functions are employed in velocity whereas bilinear shape functions are used for the pressure. Standard triangular TaylorHood element was used to construct the domain with a denser mesh in between $x=2$ and $x=3$ as shown in Fig. 2. to capture the magnitude of the higher order of magnetic gradient. The point of evaluation for each element is shown at the Fig. 3. where a triangular element is having total six nodes at the vertices and 3 nodes at the midpoint.

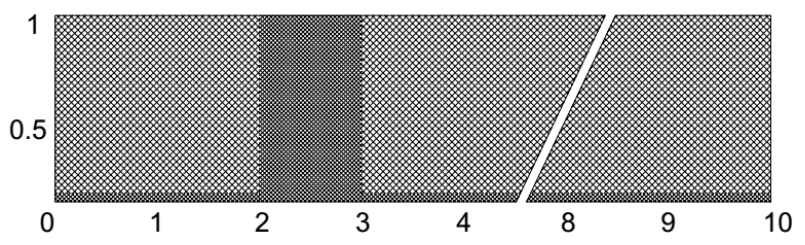

Fig. 2. Meshing of the flow domain.

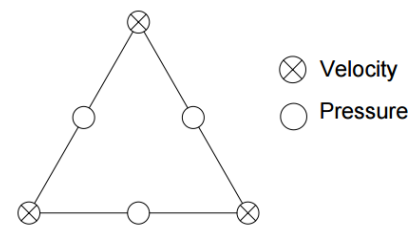

Fig. 3. Triangular Taylor-Hood element.

Non-structure grid was used to construct a nonuniform mesh. Mesh testing was conducted to ensure the grid independency of the solution. Subsequently, the total number of element used was 31,606 resulting 213,899 size of stiffness matrix. The FEM discretized system is solved iteratively with MATLAB package involving sparse solver.

\subsection{Algorithm Efficiency}

One of the main challenges in computation lies on solving massive matrix that is consuming computer resources and it can only be resolved using an efficient algorithm. Vector processing, which is a single instruction to operate multiple data gives a better performance in term of speed and memory usage. As shown in Fig. 4, different algorithm gives different efficiencies. Local imposition means impose boundary at local matrix has been found as able to execute the process more rapidly, which is 44 times faster than global imposition to complete the simulation.

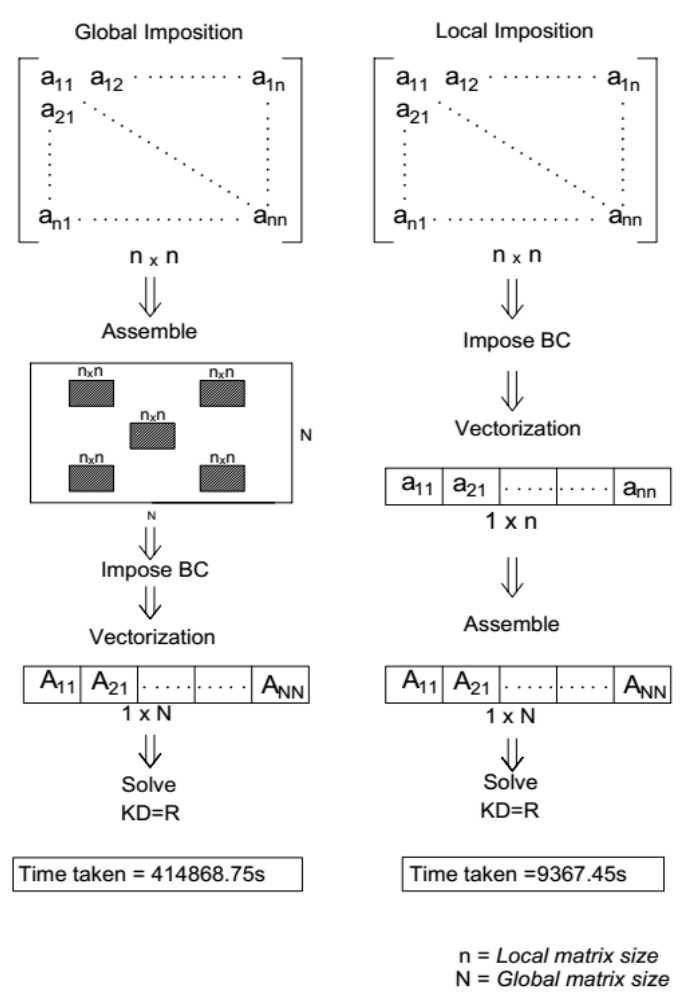

Fig. 4. Comparison of the flow model algorithms.

\section{Numerical Results and Discussion}

Table 1. Variables for Magnetic Fluid in Rectangular Channel.

\begin{tabular}{|l|c|}
\hline \multicolumn{1}{|c|}{ Variable } & Value \\
\hline Density, $\rho$ & $1050 \mathrm{~kg} / \mathrm{m}^{3}$ \\
\hline Viscosity, $\mu$ & $3.2 \mathrm{e}-3 \mathrm{~kg} /(\mathrm{ms})$ \\
\hline $\mathrm{a}$ & $2.5 \mathrm{~m}$ \\
\hline $\mathrm{b}$ & $-0.05 \mathrm{~m}$ \\
\hline Magnetization, $\mathrm{M}$ & $60 \mathrm{~A} / \mathrm{m}$ \\
\hline Reynold number, $\mathrm{Re}$ & 250 \\
\hline Eckert number, Ec & $2.456 \times 10^{-6}$ \\
\hline Prandlt number, Pr & 20 \\
\hline Temperature number, $\varepsilon$ & 8 \\
\hline Magnetic number, $\mathrm{Mn}_{\mathrm{f}}$ & 315 \\
\hline
\end{tabular}


Initially some verification calculations were performed for pure hydrodynamic case taken $\mathrm{Mn}_{\mathrm{f}}=0$ and the results are well agreed with [16]. The purpose is to assess the stability and accuracy of the finite element algorithm.

Numerical solution for magnetic fluid is verified with [12]. The dimensionless axial velocity component is shown in Fig. 5 at various magnetic field strengths with $\mathrm{Re}=250$. The velocity increases from 1.1 (2T), $1.3(4 \mathrm{~T})$, $1.43(6 \mathrm{~T})$ to $1.55(8 \mathrm{~T})$. The increment of the velocity component is due to the additional energy transport from the stress arising from magnetic strength. At the upper channel, velocity increases as the magnetic strength increases whereas at lower edge the velocity is retarded. It is also observed that recirculation zone occurred at the lower part of channel where the back flow has the minimum value of -0.20 . This physically shows that the flow has slowed down.

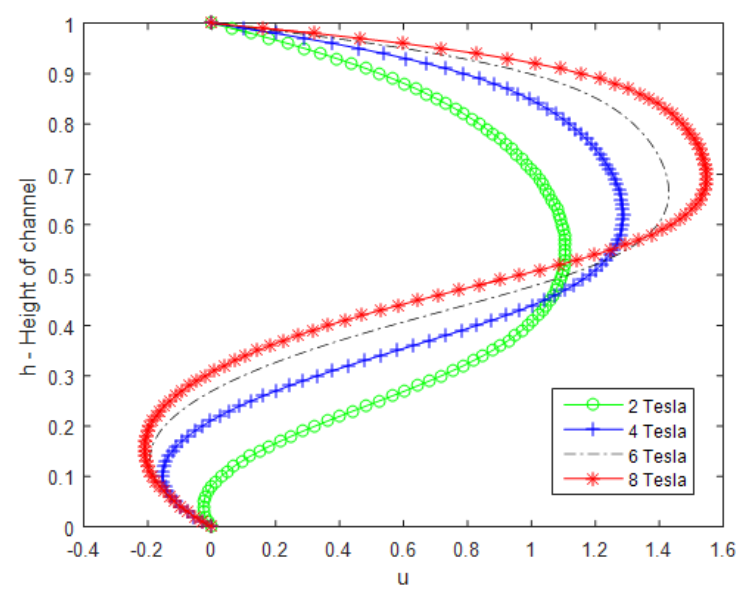

Fig. 5. Axial velocity profile in various magnetic field strengths.

Fig. 6 shows the behaviour of the temperature profile along the middle of channel. The temperature increases rapidly in the region $x=2.0$ where it is close to magnetic source and plunged drastically to its minimum value at $x=3.5$ then raised steadily. From the point $x=7.5$ onward, temperature take its entrance value as it is approaching outlet. It can be seen that, the increment of $\mathrm{Mn}_{\mathrm{f}}$ leads to greater variation of the temperature value.

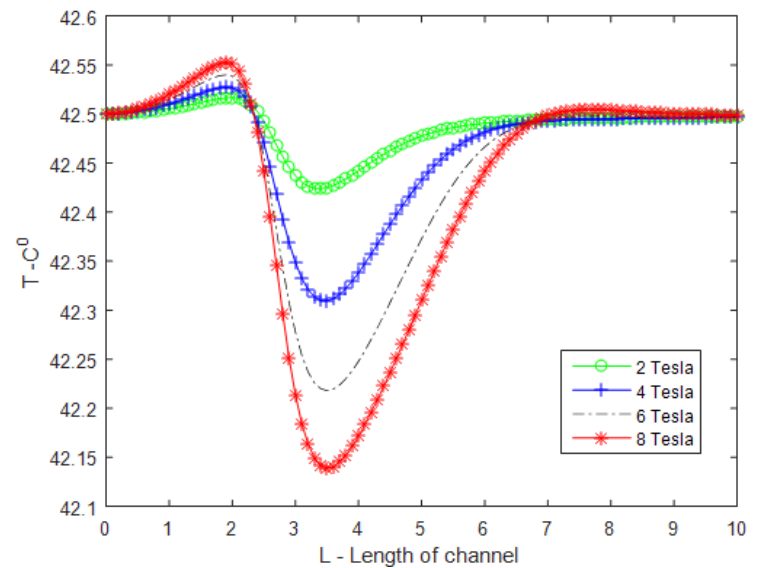

Fig. 6. Temperature profile along the middle of channel subjected to various magnetic strengths.

Fig.7 presents the velocity contours for $2 \mathrm{~T}, 4 \mathrm{~T}, 6 \mathrm{~T}$, and $8 \mathrm{~T}$ magnetic strength corresponding to the magnetic number 78, 157, 236 and 315, respectively. The vortex grows near the source and extends in length and height nearby the magnetic source as the magnetic strength increase. At the exit of channel, the velocity gained back fully developed flow.

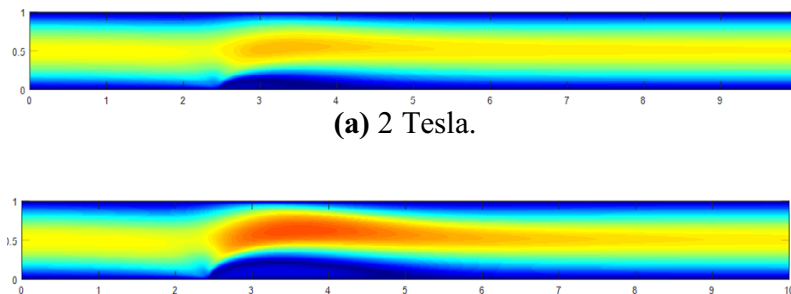

(b) 4 Tesla.

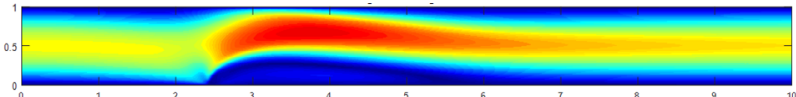

(c) 6 Tesla.

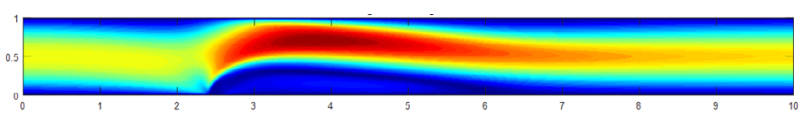

(d) 8 Tesla.

Fig. 7. Velocity contours for fluid flow model for magnetic field strength of (a) $2 \mathrm{~T}$, (b) $4 \mathrm{~T}$, (c) $6 \mathrm{~T}$ and (d) $8 \mathrm{~T}$.

\section{Concluding Remarks}

A mixed finite element formulation for magnetic fluids is successfully developed up to 8 Tesla. The results indicate the feasibility of the mixed formulation as an alternative in the modelling of the magnetic fluid flow. The outcome of this study would provide an insight into the interaction of magnetic fluid with electromagnetics which can be important in various engineering fields especially in oil and gas extraction process. 


\section{References}

1. O. A. Alomair, K. M. Matar, and Y. H. Alsaeed, "Nanofluids Application for Heavy Oil Recovery," no. October, pp. 14-16, (2014).

2. A. Roustaei, S. Saffarzadeh, and M. Mohammadi, "An evaluation of modified silica nanoparticles' efficiency in enhancing oil recovery of light and intermediate oil reservoirs," Egyptian Journal of Petroluem, vol. 22, no. 3, pp. 427-433, (2013).

3. Q. Xue, "Model for effective thermal conductivity of nanofluids," Physics Letters A, vol. 307, no. 5-6, pp. 313-317, (2003).

4. J. a. Eastman, S. U. S. Choi, S. Li, L. J. Thompson, and S. Lee, "Enhanced Thermal Conductivity through the Development of Nanofluids," $M R S$ Proc., vol. 457, pp. 3-11, (1997).

5. R. E. E. Rosensweig, "Heating magnetic fluid with alternating magnetic field," Journal of Magnetism and Magnetic Materials, vol. 252, no. 0, pp. 370374, (2002).

6. W. Lian, Y. Xuan, and Q. Li, "Characterization of miniature automatic energy transport devices based on the thermomagnetic effect," Energy Conversion and Management, vol. 50, no. 1, pp. 35-42, (2009).

7. A. Davidson, C. Huh, and S. L. Bryant, "Focused Magnetic Heating Utilizing Superparamagnetic Nanoparticles for Improved Oil Production Applications," in SPE International Oilfield Nanotechnology Conference and Exhibition, (2012).

8. F. S. D. A. Soares, M. Prodanovic, and C. Huh, "Excitable Nanoparticles for Trapped Oil Mobilization," SPE Imroved Oil Recover. Simp., no. April, pp. 1-16, (2014).

9. C. Oldenburg, S. Borglin, and G. Moridis, "Numerical simulation of ferrofluid flow for subsurface environmental engineering applications," Transport Porous Media, vol. 38 , pp. 319-344, (2000).

10. Y. Haik, V. Pai, and C. Chen, "Biomagnetic fluid dynamics," Fluid dynamics at interfaces, pp. 439452, (1999).

11. E. Tzirtzilakis and V. Loukopoulos, "Biofluid flow in a channel under the action of a uniform localized magnetic field," Computational Mechanics, vol. 36, pp. 360-374, (2005).

12. Loukonoulos. V. C.. and E. E. Tzirtzilakis. "Biomagnetic channel flow in snatiallv varving magnetic field." International .Journal of Engineering Science vol. 42, no. 5: 571-590, (2004).

13. E. Tzirtzilakis, "A mathematical model for blood flow in magnetic field," Physics of Fluids (1994present), vol. 17, p. 077103, (2005).

14. S. Kenjereš, "Numerical analysis of blood flow in realistic arteries subjected to strong non-uniform magnetic fields," International Journal of Heat and Fluid Flow, vol. 29, pp. 752-764, (2008).

15. M. Tezer-Sezgin, C. Bozkaya, and Ö. Türk, "BEM and FEM based numerical simulations for biomagnetic fluid flow," Engineering Analysis with Boundary Elements, vol. 37, pp. 1127-1135, (2013).

16. L. Q. Tang and T. T. Tsang, "A least-squares finite element method for time-dependent incompressible flows with thermal convection," International Journal for Numerical Methods in Fluids, vol. 17, pp. 271-289, (1993). 